\title{
A novel approach to the theory of Padé approximants
}

\author{
Christopher ATHORNE \\ Department of Mathematics, University of Glasgow, Glasgow G12 8QW, United Kingdom \\ E-mail: c.athorne@maths.gla.ac.uk
}

This article is a part of the special issue titled "Symmetries and Integrability of Difference Equations (SIDE VI)"

\begin{abstract}
By associating polynomials and power series expansions with $\mathfrak{s} l_{n}(\mathbb{C})$ modules we describe the theory of Padé approximants in terms of tensor products of representations and interpret their recurrence relations algebraically. The treatment links with the theory of Hirota derivatives and discrete integrable models and is generalizable to larger numbers of variables. This paper summarizes and extends the work of [2].
\end{abstract}

\section{Introduction}

The primary aim of this work is to apply the representation theory of Lie algebras to Padé approximants and to make a case, by demonstration, for the use of such methods in that area. A secondary aim is to draw together certain ideas in the field of integrable systems, namely integrable lattice equations and Hirota derivatives, under the umbrella of representation theory.

Although driven by prejudice these aims are also motivated by phenomenology. The prejudice is that visually complex algebraic expressions are significant only in the contexts of simple algebraic structures, the phenomenological observation that generic expressions for the coefficients of Padé approximants are curiously reminiscent of expressions occurring in algebraic invariant theory [11].

The paper is divided into six sections.

Firstly we review the essentials of the theory of Padé approximants and establish the notation to be used. Secondly we explain how the polynomial, rational and analytic functions are associated with covariants and invariants of the Lie algebra $\mathfrak{s} l_{2}$. In the third section we explicitly show how the Padé approximants of a given analytic function are generated by Lie algebraic operations and associated with certain highest weight representations of $\mathfrak{s} l_{2}$. The next section provides an algebraic formulation of the classical Hirota derivative and derives the recurrence relations for the highest weight vectors associated to the rational approximations. Following this we indicate how the theory is generalized to $n$ variable rational functions (multi variable Padé approximants). We claim this leads to deformed discrete $\mathrm{KdV}$ equations. 
The historical origins of Padé approximants lie within the development of continued fractions [6]. Either can be viewed as arising from 2-term recurrence relations:

$$
f_{k-1}=l_{k} f_{k}+q_{k} f_{k+1}
$$

where the coefficients $l_{k}$ and $q_{k}$ are either independent or dependent on a parameter, $x$.

If we choose $l_{k}$ to be linear in $x$ and $q_{k}$ to be quadratic then we obtain a generalization

$$
f_{k-1}(x)=\left(a_{k}+b_{k} x\right) f_{k}(x)+\left(c_{k}+d_{k} x+e_{k} x^{2}\right) f_{k+1}(x)
$$

of a recurrence relation first considered by Lagrange and leading to a sequence of $[k-1, k]$ Padé approximants. Although we will not deal directly with this relation in what follows, we can regard as motivation the following observation: that the change of variables

$$
\begin{aligned}
& x \mapsto X=\frac{\alpha x+\beta}{\gamma x+\delta} \\
& f_{k}(x) \mapsto F_{k}(X)=(\gamma x+\delta)^{k-1} f_{k}(x)
\end{aligned}
$$

acts covariantly on the recurrence relation, so that,

$$
F_{k-1}(X)=\left(A_{k}+B_{k} X\right) F_{k}(X)+\left(C_{k}+D_{k} X+E_{k} X^{2}\right) F_{k+1}(X)
$$

where the upper case coefficients, $A_{k}, \ldots E_{k}$ are simply related to the lower case, $a_{k}, \ldots e_{k}$, through the parameters $\alpha, \ldots \delta$ of the transformation.

All that follows below is a simple consequence of this covariance.

\section{Padé approximants}

In general Padé approximants are rational approximations to analytic functions (which may themselves be rational).

All functions will be defined on the projective line on which we use homogeneous coordinates $x$ and $y$. The fundamental identity for the $[n, m]$ Padé approximant of a function $f(z)$ analytic at $z=0$ is:

$$
\frac{P^{[n, m]}(x, y)}{Q^{[n, m]}(x, y)}=F^{[n, m]}(x, y)=y^{n-m} \sum_{i=0}^{\infty} f_{i}^{[n, m]} z^{i}
$$

where:

- $n$ and $m$ are positive or zero integers and $P^{[n, m]}(x, y)$ and $Q^{[n, m]}(x, y)$ homogeneous polynomials in $x$ and $y$ of degrees $n$ and $m$ respectively;

- $z=\frac{x}{y}$ is the inhomogenous variable on one coordinate chart of the projective line;

- the first $n+m+1$ Taylor coefficients of the given function $f(z)$ coincide with the first $n+m+1$ coefficients of $y^{m-n} F^{[n, m]}(x, y)$, namely $f_{0}^{[n, m]}, f_{1}^{[n, m]}, f_{2}^{[n, m]}, \ldots, f_{n+m}^{[n, m]}$. 
We will adopt the point of view that the function $F^{[n, m]}(x, y)$, the expansion of the rational approximation, is the "fundamental" object and that the coefficients

$$
f_{0}^{[n, m]}, f_{1}^{[n, m]}, f_{2}^{[n, m]}, \ldots, f_{n+m}^{[n, m]}
$$

are the "fundamental" variables in terms of which everything else will be expressed. The superfices $[n, m]$ will normally be suppressed, they being implicit in the context.

Equation (2.1) leads to an infinite number of equations linear in the coefficients of $P^{[n, m]}(x, y)$ and $Q^{[n, m]}(x, y)$. We use the following notation for these coefficients

$$
\begin{aligned}
& P^{[n, m]}(x, y)=p_{0}^{[n, m]} y^{n}+p_{1}^{[n, m]} x y^{n-1}+\ldots+p_{n}^{[n, m]} x^{n} \\
& Q^{[n, m]}(x, y)=q_{0}^{[n, m]} y^{m}+q_{1}^{[n, m]} x y^{m-1}+\ldots+q_{m}^{[n, m]} x^{m}
\end{aligned}
$$

and, as before, suppress the superfices, so that amongst the infinite set of linear equations are a special set of homogeneous equations (written here in the case that $n<m$ ),

$$
\left(\begin{array}{cccccccc}
f_{n+1} & f_{n} & f_{n-1} & \ldots & f_{0} & 0 & \ldots & 0 \\
f_{n+2} & f_{n+1} & f_{n} & \ldots & f_{1} & f_{0} & \ldots & 0 \\
\vdots & & & & & & \ddots & 0 \\
\vdots & & & & & & & f_{0} \\
\vdots & & & & & & & \vdots \\
f_{n+m+1} & f_{n+m} & \ldots & \ldots & \ldots & \ldots & \ldots & f_{n+1}
\end{array}\right)\left(\begin{array}{c}
q_{0} \\
q_{1} \\
\vdots \\
\vdots \\
\vdots \\
q_{m}
\end{array}\right)=0
$$

In fact, this linear system tells us all we need to know to construct the rational approximant and the explicit representation theory.

\section{Modules associated with functions}

Initially we define $\mathfrak{s} l_{2}$ modules spanned by monomials in $x, y, x^{-1}$ and $y^{-1} \cdot \mathfrak{s} l_{2}$ is the $\mathbb{C}$ algebra generated by

$$
\begin{aligned}
& \mathbf{e}=x \partial_{y} \\
& \mathbf{f}=y \partial_{x} \\
& \mathbf{h}=x \partial_{x}-y \partial_{y}
\end{aligned}
$$

with antisymmetric product (commutation)

$$
\begin{aligned}
& {[\mathbf{e}, \mathbf{f}]=\mathbf{h}} \\
& {[\mathbf{h}, \mathbf{e}]=2 \mathbf{e}} \\
& {[\mathbf{h}, \mathbf{f}]=-2 \mathbf{f}}
\end{aligned}
$$

We define a module $V^{[n]}$ and a submodule $V_{0}^{[n]}$ thereof as $\mathbb{C}$ spans of basis elements:

$$
\begin{aligned}
& V^{[n]}=\operatorname{Span}_{\mathbb{C}}\left\{x^{i} y^{j} \mid i, j \in \mathbb{Z}, i+j=n\right\} \\
& V_{0}^{[n]}=\operatorname{Span}_{\mathbb{C}}\left\{x^{i} y^{j} \mid i \in \mathbb{N}, j \in \mathbb{Z}, i+j=n\right\}
\end{aligned}
$$


and the notation $\mathbb{N}$ refers to the additive semigroup, so $0 \in \mathbb{N}$.

$V_{0}^{[n]}$ is a submodule of $V^{[n]}$ with a highest weight element $y \in \operatorname{ker} \mathbf{f}$.

In the case that $n \geq 0, V^{[n]}$ has a finite dimensional submodule $V_{g l o b}^{[n]}$ spanned by monomials in $x$ and $y$ of degree $n$.

Now introduce a $\mathbb{C}$-vector space

$$
F^{[n]}=\operatorname{span}_{\mathbb{C}}\left\{f_{i, j}^{[n]} \mid i, j \in \mathbb{Z}, i+j=n\right\}
$$

and a pairing

$$
\begin{aligned}
F^{[n]} \times V^{[n]} & \rightarrow \text { homogeneous Laurent series } \\
(f, v) & \mapsto \sum_{i+j=n} f_{i, j}^{[n]} x^{i} y^{j}
\end{aligned}
$$

In order to extend the $\mathfrak{s l}_{2}$ action to $F^{[n]}$ we use invariance of this pairing. Thus

$$
(\mathbf{e}(f), v)+(f, \mathbf{e}(v))=0
$$

implies

$$
\mathbf{e}\left(f_{i, j}^{[n]}\right)=-(j+1) f_{i-1, j+1}^{[n]}
$$

Similarly

$$
\begin{aligned}
& \mathbf{f}\left(f_{i, j}^{[n]}\right)=-(i+1) f_{i+1, j-1}^{[n]} \\
& \mathbf{h}\left(f_{i, j}^{[n]}\right)=(j-i) f_{i, j}^{[n]}
\end{aligned}
$$

The Laurent series represented by $(f, v)$ is thus an invariant of the Lie algebra action generalizing the approach to invariants adopted in the classical literature [11]. Because we wish to restrict attention to functions with analytic expansions the above Lie algebra action has to be restricted to a quotient module of $F^{[n]}$. There is a submodule

$$
\left(V_{0}^{[n]}\right)^{\perp}=\left\{f \in F^{[n]} \mid(f, v)=0, \forall v \in V_{0}^{[n]}\right\} \triangleleft F^{[n]}
$$

and we can define the analytic quotient module $F_{0}^{[n]}$ as the factor module

$$
F_{0}^{[n]}=F^{[n]} /\left(V_{0}^{[n]}\right)^{\perp}
$$

with the inherited $\mathfrak{s} l_{2}$ action. So now the pairing above restricts to

$$
F_{0}^{[n]} \times V_{0}^{[n]} \rightarrow \text { homogeneous series analyticat } x=0
$$

The relation (2.1) is now interpreted as a relation between invariants $P^{[n, m]}, Q^{[n, m]}$ and $F^{[n, m]}$, the linear relations $(2.4)$ are covariant under the $\mathfrak{s l}_{2}$ action and the relations between the coefficients of $P^{[n, m]}, Q^{[n, m]}$ and $F^{[n, m]}$ become expressed in terms of factors of tensor products of the analytic quotient module associated with $F^{[n, m]}$. We are effectively constructing finite dimensional modules (of dimensions $n$ and $m$ ) from an infinite dimensional module, $F_{0}^{[n]}$. 


\section{Generating the Padé table}

The immediate problems are:

- to construct $F^{[n, m]}$ from $F_{0}^{[\sigma]}$;

- to construct $q_{0}^{[n, m]}$ from $F^{[n, m]}$;

- to obtain the recurrence relations;

- to give an algebraic construction of the highest weight element associated with the $[n, m]$ approximant.

The coefficients of the analytic function $F^{[n, m]}(x, y)$ behave like a representation of the form $F^{[n-m]}$ but with a set of relations coming from an infinite set of linear equations of which the homogeneous equation (2.4) is the simplest. Letting

$$
\delta^{[n, m]}=\left|\begin{array}{cccccccc}
f_{n+1} & f_{n} & f_{n-1} & \ldots & f_{0} & 0 & \ldots & 0 \\
f_{n+2} & f_{n+1} & f_{n} & \ldots & f_{1} & f_{0} & \ldots & 0 \\
\vdots & & & & & & \ddots & 0 \\
\vdots & & & & & & & f_{0} \\
\vdots & & & & & & & \vdots \\
f_{n+m+1} & f_{n+m} & \ldots & \ldots & \ldots & \ldots & \ldots & f_{n+1}
\end{array}\right|
$$

the relations are $\delta^{[n, m]}=0$ and all other relations obtained by application of $\mathbf{f}$ to this relation.

$\delta^{[n, m]}$ is itself a highest weight element for, if not, we could obtain a relation independent of $f_{n+m+1}$ by an application of e contradicting the independence of the fundamental variables. This highest weight element thus defines a submodule of relations living inside the symmetric $m+1$-fold tensor product module,

$$
\bigodot^{m+1} F_{0}^{[\sigma]}
$$

As such it must be isomorphic to one of the $F_{0}^{[\tau]}$, for some $\tau \in \mathbb{Z}$, an identification which can be made more precise by an application of the Casimir operator

$$
\mathbf{C}=\mathbf{e f}+\mathbf{f e}+\frac{1}{2} \mathbf{h}^{2}
$$

which has eigenvalue $\frac{1}{2} \tau(\tau+2)$ on any element of $F_{0}^{[\tau]}$. It is easily evaluated on $\delta^{[n, m]}$ simply by examining the two terms of highest degree in $f_{n+1}$ and it is seen that $\delta^{[n, m]}$ is highest weight element for a submodule of $\bigodot^{m+1} F_{0}^{[\sigma]}$ isomorphic to $F_{0}^{[-(m+1)(n+m+2)]}$.

In order to construct the coefficients of the polynomials $P^{[n, m]}$ and $Q^{[n, m]}$ it is efficient to start with $q_{0, m}^{[n, m]}$. The cofactor expansion of $\delta^{[n, m]}$ dictates that the $q_{i, m-i}^{[n, m]}$ are the determinantal cofactor expressions up to an overall factor. This overall factor is easily determined by insisting that

$$
\mathbf{f}\left(q_{0, m}^{[n, m]}\right)=-q_{1, m-1}^{[n, m]}
$$


which implies an easily solvable differential equation for the factor.

In this way

$$
q_{0, m}^{[n, m]}=\left(\delta^{[n, m-1]}\right)^{-\frac{1}{n+m+1}}
$$

and all the other $q$ 's follow by application of $\mathbf{f}$. Then also

$$
p_{0, m}^{[n, m]}=f_{0} q_{0, m}^{[n, m]}
$$

and applications of $\mathbf{f}$ yield the others.

The following is an example of the procedure.

Consider the case $n=m=2, \sigma=0$. Then

$$
\begin{aligned}
\frac{p_{0} y^{2}+p_{1} x y+p_{2} x^{2}}{q_{0} y^{2}+q_{1} x y+q_{2} x^{2}} & =F^{[2,2]} \\
& =f_{0}+f_{1} \frac{x}{y}+f_{2} \frac{x^{2}}{y^{2}}+f_{3} \frac{x^{3}}{y^{3}}+f_{4} \frac{x^{4}}{y^{4}}+f_{5} \frac{x^{5}}{y^{5}}+\ldots
\end{aligned}
$$

and we consider $f_{0}, f_{1}, f_{2}, f_{3}$ and $f_{4}$ to be the fundamental variables. They satisfy a relation with $f_{5}$ :

$$
\delta^{[2,2]}=\left|\begin{array}{lll}
f_{3} & f_{2} & f_{1} \\
f_{4} & f_{3} & f_{2} \\
f_{5} & f_{4} & f_{3}
\end{array}\right|=0 .
$$

We start with

$$
\delta^{[2,1]}=\left|\begin{array}{ll}
f_{3} & f_{2} \\
f_{4} & f_{3}
\end{array}\right|
$$

and

$$
q_{0}=\left(f_{3}^{2}-f_{2} f_{4}\right)^{-\frac{1}{5}} .
$$

Successive application of $-\mathbf{f}$ and $-\frac{1}{2} \mathbf{f}$, modulo the relation $\delta^{[2,2]}=0$, yields

$$
\begin{aligned}
& q_{1}=\left(f_{3}^{2}-f_{2} f_{4}\right)^{-\frac{1}{5}}\left(f_{3} f_{1}-f_{2}^{2}\right)^{-1}\left(f_{1} f_{4}-f_{2} f_{3}\right) \\
& q_{2}=\left(f_{3}^{2}-f_{2} f_{4}\right)^{-\frac{1}{5}}\left(f_{3} f_{1}-f_{2}^{2}\right)^{-1}\left(f_{2} f_{4}-f_{3}^{2}\right)
\end{aligned}
$$

and it is easily checked that $q_{2}$ is in the kernel of $\mathbf{f}$. Likewise

$$
p_{0}=f_{0}\left(f_{3}^{2}-f_{2} f_{4}\right)^{-\frac{1}{5}}
$$

and successive application of $-\mathbf{f}$ and $-\frac{1}{2} \mathbf{f}$, modulo the relation $\delta^{[2,2]}=0$, yields

$$
\begin{aligned}
& p_{1}=\left(f_{3}^{2}-f_{2} f_{4}\right)^{-1 / 5}\left(f_{1} f_{3}-f_{2}^{2}\right)^{-1}\left(f_{1}^{2} f_{3}-f_{0} f_{1} f_{4}+f_{0} f_{2} f_{3}-f_{1} f_{2}^{2}\right) \\
& p_{2}=\left(f_{3}^{2}-f_{2} f_{4}\right)^{-1 / 5}\left(f_{1} f_{3}-f_{2}^{2}\right)^{-1}\left(f_{0} f_{2} f_{4}+2 f_{1} f_{2} f_{3}-f_{2}^{3}-f_{1}^{2} f_{4}-f_{0} f_{3}^{2}\right)
\end{aligned}
$$


and it is easily checked that $p_{2}$ is in the kernel of $\mathbf{f}$.

Although the expressions for the coefficients are algebraic rather than polynomial, it is guaranteed that a common algebraic factor cancels between the numerator and denominator of the rational function. We obtain, finally,

$F^{[2,2]}=$

$\frac{\left(f_{0} f_{1} f_{3}-f_{0} f_{2}^{2}\right) y^{2}+\left(f_{0} f_{2} f_{3}+f_{1}^{2} f_{3}-f_{0} f_{1} f_{4}-f_{1} f_{2}^{2}\right) x y+\left(f_{0} f_{2} f_{4}+2 f_{1} f_{2} f_{3}-f_{2}^{3}-f_{1}^{2} f_{4}-f_{0} f_{3}^{2}\right) x^{2}}{\left(f_{1} f_{3}-f_{2}^{2}\right) y^{2}+\left(f_{2} f_{3}-f_{1} f_{4}\right) x y+\left(f_{2} f_{4}-f_{3}^{2}\right) x^{2}}$

Thus all the information about the generic $[n, m]$ Padé approximant is contained in the determinants $\delta^{[n, m-1]}$ defining $q_{0}$ and $\delta^{[n, m]}$ defining a module of relations.

\section{Recurrence relations and Hirota derivatives}

Relations between Padé approximants labelled by different integer pairs exist and originate with Frobenius [9]. One class of such relations are due to Wynn [14] and are sometimes called the "missing relations of Frobenius". Gragg [10] summarises the situation.

There are actually a host of relations quoted in the literature which are mutually dependent. It is not altogether clear to the present author what "missing" means: certainly Frobenius was aware of five point relations. (See [9] eqn. 17.)

From the current point of view there are two points to be made. Firstly, we can establish equivalent classes of relations between the $\delta^{[n, m]}$. Viewed as a lattice function $\delta$ satisfies both four-point (Frobenius-like) and five-point (missing Frobenius-like) relations and these can be shown to be mutually consistent. Secondly, the $\delta$, being interpreted as highest weight vectors of (infinite dimensional) representations of $\mathfrak{s} l_{n}(\mathbb{C}$ ), can also be constructed using a Hirota map which is really just a slight extension of the classical Hirota derivative [12].

The relations are obtained using the determinantal identity (variously ascribed, [1]),

$$
\left.|A|\right|_{i, k} A_{l, j}|=|{ }_{i} A_{j}\left|{ }_{k} A_{l}\right|-{ }_{i} A_{l} \|_{k} A_{j} \mid
$$

where the prefixes denote omitted rows and the suffices omitted columns.

Choosing $|A|=\delta^{[n, m+1]}$ and $i=l=1, k=j=m+2$ gives

$$
\delta^{[n, m+1]} \delta^{[n, m-1]}=\delta^{[n+1, m]} \delta^{[n-1, m]}-\delta^{[n, m]^{2}}
$$

a five point relation centred on lattice site $[n, m]$.

Four point relations follow from the choices $i=n, k=1, l=1$ and $j=2$. This leads to the "lozenge" type relation:

$$
\delta^{[n, m]} \delta^{[n+1, m+2]}=\frac{1}{n+m+3} \mathbf{f}\left(\delta^{[n, m+1]}\right) \delta^{[n+1, m+1]}-\frac{1}{n+m+4} \delta^{[n, m+1]} \mathbf{f}\left(\delta^{[n+1, m+1]}\right)
$$


Similarly,

$$
\delta^{[n, m]} \delta^{[n+2, m+1]}=\frac{1}{n+m+3} \mathbf{f}\left(\delta^{[n+1, m]}\right) \delta^{[n+1, m+1]}-\frac{1}{n+m+4} \delta^{[n+1, m]} \mathbf{f}\left(\delta^{[n+1, m+1]}\right)
$$

If we consider a relation of type (5.3) between the sites with labels $(n, m-1),(n+$ $1, m),(n+1, m+1)$ and $(n, m)$ and a relation of type (5.4) between $(n-1, m),(n, m+$ $1),(n+1, m+1)$ and $(n, m)$ we may eliminate the site $(n+1, m+1)$ to leave a five-point relation between sites $(n, m-1),(n, m+1),(n-1, m),(n+1, m)$ and $(n, m)$ which is exactly the relation (5.2). This relation is, in principle, equivalent to the discrete $\mathrm{KdV}$ equation [13]

Now let us define the bilinear Hirota maps $\mathbb{E}$ and $\mathbb{F}$ acting on two fold tensor products of $\mathfrak{s} l_{n}$-modules.

$$
\begin{aligned}
& \mathbb{E}: F^{[n]} \otimes F^{[m]} \rightarrow F^{[n+1]} \otimes F^{[m+1]} \\
& \mathbb{E}\left(f_{i, j}^{[n]} \otimes f_{k, l}^{[m]}\right)=-(i+1)(l+1) f_{i+1, j}^{[n+1]} \otimes f_{k, l+1}^{[m+1]}+(j+1)(k+1) f_{i, j+1}^{[n+1]} \otimes f_{k+1, l}^{[m+1]} \\
& \mathbb{F}: F^{[n]} \otimes F^{[m]} \rightarrow F^{[n-1]} \otimes F^{[m-1]} \\
& \mathbb{F}\left(f_{i, j}^{[n]} \otimes f_{k, l}^{[m]}\right)=-f_{i-1, j}^{[n+1]} \otimes f_{k, l-1}^{[m+1]}+f_{i, j-1}^{[n+1]} \otimes f_{k-1, l}^{[m+1]}
\end{aligned}
$$

These maps have two important properties:

- They commute (intertwine) with the actions of $\mathbf{e}$ and $\mathbf{f}$;

- They themselves generate a representation of $\mathfrak{s} l_{n}$.

The intertwining property means that highest weight modules in the domain of $\mathbb{E}$ and $\mathbb{F}$ are mapped to highest weight vectors in the target space. Being highest vectors the $\delta^{[n, 1]}$ can be constructed in precisely this way, by an appropriate number of actions of $\mathbb{E}$. In fact:

$$
\begin{aligned}
\frac{1}{(2 n) !} \operatorname{Symm}\left(\mathbb{E}^{2 n}\left(f_{0,-n-2}^{[-n-2]} \otimes f_{0,-n-2}^{[-n-2]}\right)\right) & =-(n+1) !^{2}\left(\left(f_{n,-2}^{[n-2]}\right)^{2}-f_{n-1,-1}^{[n-2]} f_{n+1,-3}^{[n-2]}\right) \\
& =-(n+1) !^{2} \delta^{[n-1,1]}
\end{aligned}
$$

In order to extend this construction to third order tensor products and so to the $\delta^{[n-1,2]}$ we need to define trilinear Hirota operators:

$$
\mathbb{E}_{12}: F^{[n]} \otimes F^{[m]} \otimes F^{[p]} \rightarrow F^{[n+1]} \otimes F^{[m+1]} \otimes F^{[p]}
$$




$$
\begin{aligned}
\left.\mathbb{E}_{12}\left(f_{i, j}^{[n]} \otimes f_{k, l}^{[m]}\right) \otimes f_{r, s}^{[p]}\right)= & (i+1)(l+1) f_{i+1, j}^{[n+1]} \otimes f_{k, l+1}^{[m+1]} \otimes f_{r, s}^{[p]} \\
& -(j+1)(k+1) f_{i, j+1}^{[n+1]} \otimes f_{k+1, l}^{[m+1]} \otimes f_{r, s}^{[p]} \\
& \\
\mathbb{E}_{23}: F^{[n]} \otimes F^{[m]} \otimes F^{[p]} \rightarrow & F^{[n]} \otimes F^{[m+1]} \otimes F^{[p+1]} \\
\mathbb{E}_{23}\left(f_{i, j}^{[n]} \otimes f_{k, l}^{[m]} \otimes f_{r, s}^{[p]}\right)= & (k+1)(s+1) f_{i, j}^{[n]} \otimes f_{k+1, l}^{[m+1]} \otimes f_{r, s+1}^{[p+1]} \\
& -(l+1)(r+1) f_{i, j}^{[n]} \otimes f_{k, l+1}^{[m+1]} \otimes f_{r+1, s}^{[p+1]} \\
\mathbb{E}_{31}: F^{[n]} \otimes F^{[m]} \otimes F^{[p]} \rightarrow & F^{[n+1]} \otimes F^{[m]} \otimes F^{[p+1]} \\
\mathbb{E}_{31}\left(f_{i, j}^{[n]} \otimes f_{k, l}^{[m]} \otimes f_{r, s}^{[p]}\right)= & (r+1)(j+1) f_{i, j+1}^{[n]} \otimes f_{k, l}^{[m+1]} \otimes f_{r+1, s}^{[p+1]} \\
& -(i+1)(s+1) f_{i+1, j}^{[n]} \otimes f_{k, l}^{[m+1]} \otimes f_{r, s+1}^{[p+1]}
\end{aligned}
$$

The third order calculations are time consuming even using MAPLE but the following holds:

$$
\operatorname{Symm}\left(\left(\mathbb{E}_{12} \mathbb{E}_{23} \mathbb{E}_{31}\right)^{n}\left(f_{0,-n-3}^{[-n-3]} \otimes f_{0,-n-3}^{[-n-3]} \otimes f_{0,-n-3}^{[-n-3]}\right)=K_{n} \delta^{[n-1,2]}\right.
$$

where the $K_{n}$ are constants. We give an argument for this form in a moment.

$\mathbb{E}_{i j}$ can be generalized to $m+1$-fold tensor products in the obvious way. A relation of the following kind is then conjectured:

$$
\operatorname{Symm}\left(\left(\mathbb{E}_{12} \mathbb{E}_{23} \ldots \mathbb{E}_{m m+1} \mathbb{E}_{m+11}\right)^{n}\left(\bigotimes^{m+1} f_{0,-n-m-1}^{[-n-m-1]}\right)=K_{n}^{m} \delta^{[n-1, m]}\right.
$$

That this is correct can be argued using a Casimir calculation. Firstly each position label $1,2, \ldots m+1$ occurs twice in the product $\mathbb{E}_{12} \mathbb{E}_{23} \ldots \mathbb{E}_{m m+1} \mathbb{E}_{m+11}$ so that each term in the tensor product is raised from signature $-n-m-1$ by $2 n$ to $n-m-1$ which is correct for the definition of $\delta^{[n-1, m]}$. Further, since the $\mathbb{E}_{i j}$ commute with the $\mathfrak{s} l_{2}$ action the eigenvalue of the Casimir on the left hand side of (5.15) is the same as that on the highest weight vector $\otimes^{m+1} f_{0,-n-m-1}^{[-n-m-1]}$, which is easily checked to be

$$
\frac{1}{2}(m+1)(-n-m-1)((m+1)(-n-m-1)+2) .
$$

But this is equal to the known value found earlier for the module $F_{0}^{[-(m+1)(n+m+1)]}$ for which $\delta^{[n-1, m]}$ is precisely the highest weight vector.

As a prescription for calculating the $\delta$ 's this is exceedingly inefficient given that the determinant form is easy but from a theoretical point of view it emphasizes that all the objects of the theory of Padé approximants have their origin in the representation theory. It also demonstrates that the constraints of the form $\delta^{[n, m]}=0$ are interpretable as multilinear Hirota-type equations: the members of the family of rational functions of numerator degree $n$ and denominator degree $m$ each satisfy the same nonlinear differential equation of order $n+m+1$ which is Hirota multi-linearizable. 


\section{Modules and $n$-variable approximants}

Suppose now we have a function analytic and homogeneous in $p$ variables $x_{1}, x_{2}, \ldots, x_{p}$ and we wish to approximate it by a ratio of homogeneous polynomials in the same variables of degrees $n$ and $m$. This will give rise to $p$-Padé approximants which have been discussed in a classical manner by Cuyt [8]. Here we will indicate how the above treatment extends to these cases. We can write:

$$
\frac{P^{[n, m]}\left(x_{1}, x_{2}, \ldots x_{p}\right)}{Q^{[n, m]}\left(x_{1}, x_{2}, \ldots x_{p}\right)}=F^{[n, m]}\left(x_{1}, x_{1}, \ldots x_{p}\right)=\sum_{i_{1}+\ldots i_{p}=n-m}^{\infty} f_{i_{1} i_{2} \ldots i_{p}}^{[n, m]} x_{1}^{i_{1}} x_{2}^{i_{2}} \ldots x_{p}^{i_{p}}
$$

The same kinds of consideration apply as before except that now the Lie algebra $\mathfrak{s} l_{n}(\mathbb{C})$ is acting on variables and coefficients. This can be taken to be generated by elements

$$
\mathbf{e}_{i j}=x_{i} \partial_{x_{j}} \quad i \neq j=1 \ldots p
$$

whose (dual) action on the $f_{k_{1} k_{2} \ldots k_{p}}^{[n, m]}$ is

$$
\mathbf{e}_{i j}\left(f_{k_{1} k_{2} \ldots k_{p}}^{[n, m]}\right)=-\left(k_{j}+1\right) f_{k_{1} \ldots k_{i}-1 \ldots k_{j}+1 \ldots k_{p}}^{[n, m]} \quad i \neq j=0 \ldots p
$$

Again the coefficients of $f$ form an infinite dimensional representation of $\mathfrak{s} l_{n}$, those of the polynomials form finite dimensional representations. A highest weight vector in any such representation is defined as belonging to the kernel of a Borel (maximal, nilpotent) subalgebra of $\mathfrak{s} l_{n}$.

The Hirota maps are simple generalizations of those for $\mathfrak{s} l_{2}$ but there are now $n-1$ of each kind, up to linear dependence, for the two fold tensor product:

$$
\begin{aligned}
\mathbb{E}^{k l}: F^{[n]} \otimes F^{[m] \rightarrow} & F^{[n+1]} \otimes F^{[m+1]} \\
\mathbb{E}^{k l}\left(f_{i_{1} \ldots i_{p}}^{[n]} \otimes f_{j_{1} \ldots j_{p}}^{[m]}\right)= & -\left(i_{k}+1\right)\left(j_{l}+1\right) f_{i_{1} \ldots i_{k}+1 \ldots i_{p}}^{[n+1]} \otimes f_{j_{1} \ldots j_{l}+1 \ldots j_{p}}^{[m+1]} \\
& +\left(i_{l}+1\right)\left(j_{k}+1\right) f_{i_{1} \ldots i_{l}+1 \ldots i_{p}}^{[n+1]} \otimes f_{j_{1} \ldots j_{k}+1 \ldots j_{p}}^{[m+1]} \\
\mathbb{F}^{k l}: F^{[n]} \otimes F^{[m]} \rightarrow & F^{[n-1]} \otimes F^{[m-1]} \\
\mathbb{F}^{k l}\left(f_{i_{1} \ldots i_{p}}^{[n]} \otimes f_{j_{1} \ldots j_{p}}^{[m]}\right)= & -f_{i_{1} \ldots i_{k}-1 \ldots i_{p}}^{[n-1]} \otimes f_{j_{1} \ldots j_{l}-1 \ldots j_{p}}^{[m-1]}+f_{i_{1} \ldots i_{l}-1 \ldots i_{p}}^{[n-1]} \otimes f_{j_{1} \ldots j_{k}-1 \ldots j_{p}}^{[m-1]}
\end{aligned}
$$

Details of the representation theory can be found in [5].

To illustrate the issues we discuss the situation for $n=3$, with variables $x_{1}, x_{2}$ and $x_{3}$ and take the specific instance of a $[1,2]$ approximant.

Consider

$$
\frac{p_{100} x_{1}+p_{010} x_{2}+p_{100} x_{3}}{q_{200} x_{1}^{2}+q_{110} x_{1} x_{2}+q_{020} x_{2}^{2}+q_{011} x_{2} x_{3}+q_{002} x_{3}^{2}+q_{101} x_{1} x_{3}}=\sum_{i_{1}+i_{2}+i_{3}=-1} f_{i j k} x_{1}^{i_{1}} x_{2}^{i_{2}} x_{3}^{i_{3}}
$$


We focus on the dependence on one variable, say $x_{3}$ and write this as:

$$
\frac{P_{1}+P_{0} x_{3}}{Q_{2}+Q_{1} x_{3}+Q_{0} x_{3}^{2}}=F^{[-1]}+F^{[-2]} x_{3}+F^{[-3]} x_{3}^{2}+F^{[-4]} x_{3}^{3}+\ldots
$$

where each $P_{i}, Q_{i}$ and $F^{[i]}$ is homogeneous of degree $i$ in $x_{1}$ and $x_{2}$. This relation implies $\left(x_{3}=0\right)$,

$$
P_{1}=Q_{2} F^{[-1]}
$$

from which all others can be generated by applications of $\mathbf{e}_{13}$ or $\mathbf{e}_{23}$. The actions of these operators on the $P$ 's, $Q$ 's and $F$ 's is, for $i=1,2$,

$$
\begin{aligned}
& \mathbf{e}_{i 3}\left(P_{1}\right)=x_{i} P_{0} \quad \mathbf{e}_{i 3}\left(P_{0}\right)=0 \\
& \mathbf{e}_{i 3}\left(Q_{2}\right)=x_{i} Q_{1} \quad \mathbf{e}_{i 3}\left(Q_{1}\right)=2 x_{i} Q_{0} \quad \mathbf{e}_{i 3}\left(Q_{0}\right)=0 \\
& \left.\mathbf{e}_{i 3}\left(F^{[} p\right]\right)=-p x_{i} F^{[p-1]}
\end{aligned}
$$

Thus, applying either $\mathbf{e}_{13}$ or $\mathbf{e}_{23}$ to (6.9) yields (after division by common factors) the chain of further relations

$$
\begin{aligned}
& P_{0}=Q_{1} F^{[-1]}+Q_{2} F^{[-2]} \\
& 0=Q_{0} F^{[-1]}+Q_{1} F^{[-2]}+Q_{2} F^{[-3]}
\end{aligned}
$$

and, for all $p>1$,

$$
0=Q_{0} F^{[-p]}+Q_{1} F^{[-p-1]}+Q_{2} F^{[-p-2]}
$$

In this way a sequence of basic relations holds amongst the $F^{[p]}$ of which the simplest is

$$
\left|\begin{array}{lll}
F^{[-3]} & F^{[-2]} & F^{[-1]} \\
F^{[-4]} & F^{[-3]} & F^{[-2]} \\
F^{[-5]} & F^{[-4]} & F^{[-3]}
\end{array}\right|=0
$$

the others being generated using the $e_{i 3}$. This relation is analogous to the $\delta$ constraint of the two variable case except that it is, by virtue of the $F$ 's being functions of $x_{1}$ and $x_{2}$ a set of constraints on the coefficients of (6.7). Also the entries $F^{[p]}$ correspond to different representations of $\mathfrak{s} l_{2}$ for each $p$.

Each of the F's is, of course, an analytic expansion of a rational function, e.g.

$$
\begin{aligned}
& F^{[-1]}=\frac{P_{1}}{Q_{2}} \\
& F^{[-2]}=\frac{P_{0} Q_{2}-P_{1} Q_{1}}{Q_{2}^{2}}
\end{aligned}
$$

etc. each of these is treated exactly as in the two variable case using the $\mathfrak{s} l_{2}$ representation generated by $\mathbf{e}_{12}$ and $\mathbf{e}_{21}$, the $\mathbf{e}$ and $\mathbf{f}$ of earlier sections. 
Define, analogously to the $\delta^{[n, m]}$ the determinants

$$
\Delta^{[-n,-m]}=\left|\begin{array}{ccccc}
F^{[-n-1]} & F^{[-n]} & F^{[-n+1]} & \ldots & F^{[-n+m+1]} \\
F^{[-n-2]} & F^{[-n-1]} & F^{[-n]} & \ldots & \\
\vdots & & & & \\
F^{[-n-m-1]} & F^{[-n-m]} & \ldots & &
\end{array}\right| .
$$

Such objects will satisfy four- and five-point relations of exactly the same algebraic form as the $\delta^{[n, m]}$. Because the only difference is that the entries now depend on extra variables (effectively one variable by homogeneity) they amount to deformations of the earlier relations, that is a deformation of the discrete $\mathrm{KdV}$ etc. Thus each $F^{[-n]}$ can be taken to have an expansion in $x_{1}$ and $x_{2}$ analytic, say, at $x_{1}=0$ of the form,

$$
F^{[-n]}=f_{0,-n}^{[-n]} x_{2}^{-n}+f_{1,-n-1}^{[-n]} x_{1} x_{2}^{-n-1}+f_{2,-n-2}^{[-n]} x_{1}^{2} x_{2}^{-n-2}+\ldots
$$

The four- and five-point relations on the $\Delta$ 's will have similar expansions giving rise to lattice relations in three integer variables. Because all the constructions are entirely analogous to the two variable case one may believe that the integrability properties carry over to such higher dimensional deformations.

\section{Conclusions and further work}

We have demonstrated in this paper the simple way that the expressions arising in the theory of Padé approximants can be viewed as consequences of representation theory for $\mathfrak{s} l_{n}$. This provides an abstract underpinning for the combinatorial and recurrence aspects of the theory. It would be instructive to formulate other developments of the Padé theory from this point of view.

Clearly it is also important to follow up the discrete $\mathrm{KdV}$ deformations arising in the $p$-variable case.

A number of other avenues are open for study.

Firstly there are relations between the continued fraction expansions of special functions and the symmetries of the differential equations they satisfy [7]. These should be described in similar terms to the general Padé approximant but under some specialization.

Secondly, it is possible to modify the treatment to $\mathfrak{s} l_{2 q}$ and to create quantum Padé approximants in noncommutative variables $x$ and $y$. In a formal sense this is quite straightforward but a $q$-Hirota map has still to be found to complete the analogy with the classical case.

Acknowledgments. I would like to thank the Royal Society of London for funding my attendance at the conference SIDE VI held in Helsinki in 2004 and Frank Nijhoff, Vasilios Papageorgiou and Sergei Tsarev for conversations related to this work.

\section{References}

[1] Aitken A C, Determinants and Matrices, 9th Ed., Oliver and Boyd (1959)

[2] Athorne C, The representation theory of Padé approximants, J. Phys. A: Math. Gen. 37 (2004) $7699-7710$ 
[3] Athorne C, Algebraic Hirota Derivatives, Phys. Lett. A 256 (1999) 20-24

[4] Athorne C, Invariant theory and Hirota Derivatives, Glasg. Math. J. 43A (2001) 1-8

[5] Athorne C, Algebraic Hirota Maps, NATO ARW proceedings, Elba 2002.

[6] Berezinski - The long history of continued fractions and Padé approximants, in Padé Approximation and its Applications, pp. 1-27, Amsterdam 1980, Ed. M.G. de Bruin and H. van Rossum, Lecture Notes in Mathematics 888, Springer 1981.

[7] Boyer C P and Miller W Jnr, A Relationship between Lie Theory anf Continued Fraction Expansions for Special Functions, in Padé and Rational Approximation, Ed. Saff, E.B. and Varga, R.S., (1977) Academic Press.

[8] Cuyt, A A M, The QD-algorithm and Multivariate Padé-Approximants, Numer. Math. 42 (1983) pp. 259-269

[9] Frobenius G and Stickelberger L., Über die Addition und Multiplication der elliptischen Functionen, Jour. für die reine und angewandte Mathematik 88 (1880) pp. 146-184

[10] Gragg W B, The Pade Table and Its Relation to Certain Algorithms of Numerical Analysis, SIAM Review, 14 (1972) 1-62.

[11] Hilbert D, Theory of Algebraic Invariants, CUP (1993)

[12] Hirota R, Bilinearization of soliton equations, J. Phys. Soc. Japan, 51 (1982) pp. 323-331.

[13] Papageorgiou V, Grammaticos B and Ramani A, Integrable lattices and convergence acceleration algorithms Phys. Lett. A179 (1993) 111-115

[14] Wynn P, Upon systems of recursions which obtain among the quotients of the Padé table, Numer. Math. 8 (1966) pp. 246-269. 